\title{
Trophic mechanisms for exercise-induced stress resilience: potential role of interactions between BDNF and galanin
}

\author{
Philip V. Holmes* \\ Neuroscience Program, Psychology Department, Biomedical and Health Sciences Institute, The University of Georgia, Athens, GA, USA
}

Edited by:

Felipe Schuch, Hospital de Clínicas de Porto Alegre, Brazil

\section{Reviewed by:}

Tariq Munshi, Queen's University, Canada

Meera Balasubramaniam, NYU

Langone Medical Center, USA

*Correspondence:

Philip V. Holmes, Neuroscience Program, Biomedical and Health Sciences Institute, Psychology Department, The University of

Georgia, Athens, GA 30602, USA e-mail:pvholmes@uga.edu
Current concepts of the neurobiology of stress-related disorders, such as anxiety and depression emphasize disruptions in neural plasticity and neurotrophins. The potent trophic actions of exercise, therefore, represent not only an effective means for prevention and treatment of these disorders, they also afford the opportunity to employ exercise paradigms as a basic research tool to uncover the neurobiological mechanisms underlying these disorders. Novel approaches to studying stress-related disorders focus increasingly on trophic factor signaling in corticolimbic circuits that both mediate and regulate cognitive, behavioral, and physiological responses to deleterious stress. Recent evidence demonstrates that the neural plasticity supported by these trophic mechanisms is vital for establishing and maintaining resilience to stress. Therapeutic interventions that promote these mechanisms, be they pharmacological, behavioral, or environmental, may therefore prevent or reverse stress-related mental illness by enhancing resilience. The present paper will provide an overview of trophic mechanisms responsible for the enhancement of resilience by voluntary exercise with an emphasis on brain-derived neurotrophic factor, galanin, and interactions between these two trophic factors.

\section{TROPHIC MECHANISMS FOR EXERCISE-INDUCED STRESS} RESILIENCE: POTENTIAL ROLE OF INTERACTIONS BETWEEN BDNF AND GALANIN

Clinical studies convincingly establish the efficacy of exercise in the treatment of anxiety and affective disorders (1-3). However, understanding the neurobiological mechanisms responsible for these beneficial effects, a goal that necessitates the application of rodent models, remains a challenge. Since deleterious stress lies at the core of anxiety and depression, understanding the neurobiological impacts of stress, and the mechanisms for adapting to it will significantly enhance the development of more effective prevention and treatment. Recent advances in exercise neuroscience using rodent models have shed new light on the neural mechanisms by which physical activity produces long-term adaptations in brain circuits implicated in anxiety and depressive disorders. This literature reveals that the most significant impact of exercise on stress may not pertain to regulating transient states occurring in the presence of the stressor, but rather on moderating the long-term impact that acute stress may incur on subsequent stress events. The key benefit of exercise may thus involve the promotion of stress resilience.

The present paper will focus on stress resilience as a fundamental phenomenon underlying the beneficial effects of exercise, and it will briefly review the neurotrophic hypothesis of stress resilience. Two trophic mechanisms that may be involved in the stress resilience will be examined; one involving the widely studied brain-derived neurotrophic factor (BDNF) system and another based on the lesser-known trophic actions of the peptide galanin. The purpose of this comparison will be to point out a critical area for future investigation, which should aim to uncover how different trophic mechanisms may interact to promote optimal neural function.

\section{EFFECTS OF EXERCISE IN RODENT MODELS OF DEPRESSION AND ANXIETY AND THEIR IMPLICATIONS FOR STRESS RESILIENCE}

Though exercise represents an effective intervention for stressrelated disorders in humans, evidence for its antidepressant and anxiolytic efficacy in rodent behavioral models is surprisingly inconclusive. In recent, comprehensive reviews of the anxiolytic and antidepressant effects of exercise in rodent models a common theme emerges, in which that exercise produces mixed and conflicting effects in standard rodent tests of anxiety and depression (4-6). Much of the conflicting evidence derives from the species or strain used as subjects, duration, and mode of exercise employed as the independent variable, and the selection of model that serves as the dependent measure of depressive- or anxiety-like behavior. Experiments employing forced modes of exercise, such as treadmill or swimming, introduce the confounding factor of stressor exposure on subsequent measures of stress-related behavior and are thus difficult to interpret. Furthermore, rodent paradigms that putatively model depressive-like behavior are plagued by questions of validity, and the most widely employed "models," such as the forced swim or tail suspension tests, are better characterized as bioassays or screens for pharmacological manipulations with potential antidepressant activity (7). A particularly problematic aspect of these models is that they are too often assumed to represent the chronic, self-perpetuating nature of major depression by assessing a "snapshot" of spontaneous responsiveness to an acute stressor when no predisposing factors have been manipulated. 
They also assume that antidepressant actions may be observed in a healthy subject. It is with these caveats in mind that one must cautiously interpret the reports that exercise may exert either antidepressant-like, "pro-depressive," or no effects in the forced swim or similar tests $(5,8,9)$.

Similar problems of interpretation arise when critically examining exercise effects in anxiety models. When such models are applied as a single measure of spontaneous behavior in the presence of the mild stress associated with standard tests, it is difficult to ascertain whether increased responding reflects behavior reminiscent of anxiety or, rather, adaptive coping to the exigencies of the stressor. Both interpretations have been proposed in previous studies, and exercise has been variously characterized that has anxiogenic, anxiolytic, or without effect (4-6). This range of contradictory results is evident even when focusing on a single model, the elevated plus-maze. After systematically reviewing this literature, we have proposed that understanding the effects of exercise hinge upon examination of the longitudinal impact of stress exposure on subsequent responding (6). We have thus shown that the anxiolytic-like effects of exercise are only consistently observed in standard models of anxiety when rats have been previously exposed to a different type of stressor. Exercise, thus appears to promote resilience to stress.

\section{TROPHIC MECHANISIMS IN STRESS RESILIENCE}

Emerging concepts of the neurobiology of stress-related disorders, such as anxiety and depression emphasize disruptions in neural plasticity and trophic mechanisms $(10,11)$. Current research on the neurobiology of stress, thus, increasingly involves measures of neurogenesis, dendritic arborization, dendritic spine maintenance, synaptogenesis, and other forms of plasticity. This new perspective on the neurobiological basis of anxiety and depressive disorders is superseding traditional explanations that emphasize dysfunction in monoaminergic transmission as the primary etiological mechanism. The focus is thus shifting to the role of disruptions in trophic factor signaling, especially in corticolimbic circuits that mediate and/or regulate cognitive, behavioral, and physiological responses to stress. Recent evidence demonstrates that the neural plasticity supported by these trophic mechanisms is vital for establishing and maintaining stress resilience $(12,13)$. Stress-induced atrophy of the hippocampus has been linked to decreased resilience in clinical populations and rodent models (11, 14-16). Maintenance of plasticity in the medial prefrontal cortex (mPFC) also plays a crucial role in stress resilience, and disturbances in this plasticity are linked to the pathophysiology of depression and anxiety (17-21). The translational value of this new perspective on trophic mechanisms will be the identification and development of therapeutic interventions that enhance resiliencepromoting plasticity, be they pharmacological, behavioral, or environmental.

\section{ROLE OF EXERCISE-INDUCED REGULATION OF} HIPPOCAMPAL BDNF IN ANXIETY AND DEPRESSION

The prominence of trophic mechanisms in determining the pathophysiology of anxiety and depression compels further examination of the profound influence exercise exerts on trophic factor expression. Much work already has demonstrated the capacity for exercise to induce a variety of trophic factors in the brain and periphery, such as BDNF, insulin-like growth factor (IGF-1), vascular endothelial growth factor (VEGF), and the inflammatory protein VGF (11), but most of this previous research has focused on BDNF signaling in the hippocampus $(11,22-24)$.

Brain-derived neurotrophic factor is a member of the neurotrophin family, a group of structurally related peptide growth factors that signal through TrkB receptors (22). BDNF is directly implicated in various forms of hippocampal plasticity induced by exercise $(25,26)$, leading many researchers to hypothesize that this action may mediate antidepressant and anxiolytic effects of exercise $(11,15,23)$. This hypothesis is supported by numerous findings of relationships between exercise, BDNF, hippocampal plasticity, and antidepressant actions. For example, exercise consistently elevates BDNF expression in the hippocampus $(23,27$, 28) and potentiates the antidepressant activity of antidepressant drugs in the forced swim test [(9); though c.f. (8)]. Antidepressant effects of exercise in mice are eliminated by BDNF knockout and inhibition of MAPK signaling, an intracellular mediator of BDNF (29). Exercise also reliably enhances hippocampal neurogenesis (30). This evidence has provided the foundation for the hypothesis that the beneficial effects of exercise are mediated specifically by BDNF-induced neurogenesis in the hippocampus. This hypothesis is an extension, what is generally referred to as the "neurogenesis hypothesis of affective disorders." Some reports from the exercise literature support this hypothesis (11, 25 ), though systematic reviews of studies linking hippocampal BDNF to antidepressant actions in humans and rodent models found many disassociations between BDNF and depression (31, 32 ). With respect to neurogenesis specifically, exercise-induced hippocampal neurogenesis is associated with increased spontaneous anxiety-like behavior, an effect that is reversed after irradiation, which effectively eliminates neurogenesis $(33,34)$. As described above, the discrepancies in the literature may relate to whether the behavioral paradigm measures acute stress reactivity or the longitudinal impact of stress (i.e., resilience). They may also depend on the nature of trophic factor-mediated plasticity in the hippocampus. In addition to its neurogenic effects, exercise induces other forms of plasticity in hippocampal neurons, such as alterations in dendritic spine architecture (23), which must be considered in accounting for antidepressant or anxiolytic effects.

The studies presented above establish links between exercise, BDNF, and antidepressant actions. However, whether BDNFdependent actions of exercise in the hippocampus reflect antidepressant effects per se or some underlying process such as stress resilience is not clear. Hippocampal administration of BDNF does not reverse the exaggerated stress response exhibited by rats previously exposed to uncontrollable stress, and a pharmacological manipulation that reduces hippocampal BDNF does not exacerbate the effects of stress on subsequent stress responses (35). These findings suggest that BDNF signaling in the hippocampus does not have a generalized influence on stress resilience. 


\section{POTENTIAL ROLE OF EXERCISE-INDUCED REGULATION OF LOCUS COERULEUS GALANIN IN STRESS RESILIENCE}

As discussed above, exercise increases the expression of a wide range of trophic factors throughout the brain. A fuller understanding of how trophic mechanisms are involved in the beneficial effects of exercise on stress-related behaviors, therefore, requires expanding the scope beyond BDNF in the hippocampus and examining other trophic systems. Galanin is a neuromodulatory peptide and trophic factor that exerts multiple effects through its interaction with specific $G$ protein-coupled receptor subtypes designated GalR1, GalR2, and GalR3 $(36,37)$. Both galanin and its receptors are widely distributed in several brain systems, and galanin signaling thus impacts a variety of cognitive, behavioral, and endocrine functions. Some of the highest concentrations of galanin are found in the locus coeruleus (LC), where galanin is colocalized in over $80 \%$ of noradrenergic neurons (38). Galanin-containing LC neurons extensively innervate the mPFC, where galanin-immunoreactive terminals and GalR2 receptors are present in relatively high densities (39-41).

Research from my laboratory has repeatedly shown that 3 weeks of voluntary exercise elevates galanin gene expression in the LC in a running distance-dependent fashion (42-44). We have also shown that exercise exerts antidepressant effects in chronic models of depression (45), and chronic antidepressant treatment elevates galanin mRNA in the LC similarly to exercise (46). This evidence raises the obvious question of whether galanin exerts antidepressant and/or anxiolytic effects. Reviews of the extensive literature on the effects of galanin on anxiety- and depression-related behaviors show that galanin's effects on these behaviors, like those of exercise, are mixed and conflicting (47). Most of these previous experiments involved acute, central administration of galanin receptor ligands with subsequent tests of spontaneous behavior in standard models. With regard to galanin/GalR1/GalR2 transgenic mice, the findings are even more conflicting with predominantly negative findings in most standard anxiety and depression models (47).

Despite these confusing results, the behavioral evidence is clearer in experiments that examined galanin's influence on the longitudinal impact of stress. For example Lu et al. (48) reported no effect of GalR2 knockout on spontaneous behaviors such as elevated plus-maze, light/dark transition, forced swim, or tail suspension tests. However, GalR2 knock-out mice exhibited increased susceptibility to the repeated stress employed in a "learned helplessness"-like paradigm compared to wild type mice. Unlike the other tests, "learned helplessness" paradigms examine the impact of uncontrollable stress exposure on subsequent responding to repeated stressors. Loss of GalR2 led to a susceptible phenotype in this paradigm, suggesting that galanin signaling through GalR2 is necessary for resilience. Further supporting a specific role for GalR2 in resilience, transgenic overexpression of GalR2 in several frontocortical areas, including mPFC, was found to decrease immobility in a version of the forced swim test that involved pre-exposure to swim stress on the previous day (49). In contrast, GalR2 overexpression had no effect on elevated plus-maze or novel open field exploration in mice not previously exposed to stress. The behavioral literature thus points to functions for galanin beyond neuromodulatory effects. Galanin and GalR2 in particular, are evidently involved in protecting against the lasting consequences of an acute stress event by diminishing reactivity to subsequent stressors rather than by modulating reactivity to the acute stress event itself. This long-term protection suggests a role for galanin in the plasticity underlying resilience.

Galanin is well positioned as a trophic factor expressed in high concentrations in the LC to modify neural architecture in stress-responsive targets such as the mPFC. As described above, maintenance of dendritic spines in the MPFC may provide a cellular mechanism for resilience. Recent studies of galanin have revealed specific actions on neurite dynamics mediated by activation of GalR2 $(50,51)$. GalR2-mediated activation of Gq/11 influences multiple downstream targets, which includes inhibition of the RhoA GTPase. Galanin thus promotes neurite formation in neuronal cultures through a pathway involving GalR2-mediated inhibition of RhoA-ROCK signaling through LIMK, with subsequent activation of the actin-binding protein cofilin (51). Stress is associated with excess activation of RhoA, which leads to a reduction in spine densities (52). GalR2-mediated inhibition of RhoAROCK signaling thus provides a hypothetical mechanism for galanin-mediated protection against stress-induced spine atrophy. GalR2-signaling may also stabilize neurites via maintenance of microtubule integrity by promoting aggregation of microtubuleassociated protein 2 (MAP2) and $\beta$-tubulin (53), a process that may also involve inhibition of RhoA (54). These actions of galanin represent candidate mechanisms for protection against dendritic spine atrophy induced by stress.

\section{POTENTIAL INTERACTIONS BETWEEN BDNF AND GALANIN IN OPTIMIZING NEURONAL FUNCTION}

Though BDNF and galanin share common neurotrophic functions, focusing on how the systems interact provides a new approach to understanding neural plasticity. Intracellular signaling of BDNF and galanin converges in many pathways, but their mutual influence on MAP2 may reflect complementary mechanisms to promote spine maintenance during the physiological challenges imposed by stress at the cellular level. TrkB-mediated BDNF signaling promotes the expression of MAP2 and microtubule assembly (54), whereas GalR2-signaling may be more involved in maintaining microtubule assemblies by inhibiting MAP2 phosphorylation, as described above. The two trophic systems may thus mutually maintain microtubule integrity, but through distinct mechanisms. Conversely, BDNF and galanin signaling pathways diverge in the area of coflin activation. Binding of BDNF to TrkB activates a LIMK through a RAC1 pathway, which ultimately leads to the inactivation of cofilin (54). That GalR2signaling leads to the activation of cofilin through inhibition of LIMK suggests that BDNF and galanin may exert counterregulatory influences in their mutual function to promote spine maintenance.

Counter-regulatory interactions between galanin and BDNF are also evident at the systems level. Left unchecked, exerciseinduced elevations in BDNF may lead to state of neuronal hyperexcitability (22). Exercise induces a long-term enhancement of glutamatergic activity through upregulation of NMDA receptors (30). Additionally, enhanced transmission through AMPA receptors promotes $\mathrm{Ca}^{++}$-mediated $\mathrm{BDNF}$ release and signaling through TrkB $(11,54)$. The resulting BDNF-mediated effects on 
synaptic plasticity may further enhance excitatory glutamatergic transmission. This positive feedback loop between BDNF and glutamatergic activities accounts for the increased seizure vulnerability and excitotoxicity seen following experimental manipulations that enhance BDNF function, particularly in the hippocampus (55-57). This potential state of hyperexcitability following BDNF upregulation may also account for the finding of increased vulnerability to kainic acid-induced excitotoxicity following direct injection into the hippocampus of anesthetized rats that had undergone several weeks of exercise (58). In contrast to this finding in anesthetized rats, we have shown decreased vulnerability to kainic acid-induced seizures in awake, freely behaving rats (59). The galanin receptor antagonist M40 blocked this exerciseinduced protection against seizures. Taken together, the evidence suggests that under normal physiological conditions, the potential state of hippocampal hyperexcitability induced by elevated BDNF may be regulated by the galanin system. The anticonvulsant and neuroprotective properties of both endogenous and exogenous galanin are well established $(50,60,61)$. The dense innervation of the hippocampus by galaninergic projections originating from the LC (62), the presence of galanin receptors in this structure (63), and the increase in galanin with exercise (42-44), all point to this system as a regulatory mechanism controlling the deleterious consequences of exercise-induced upregulation of BDNF.

\section{CONCLUSION}

The trophic influences of exercise, which are mediated by a wide range of neural and humoral factors, are well known. This body of knowledge fits well with new concepts of anxiety and depression as they relate to disturbances in neuroplasticity, and it reveals a compelling neurobiological mechanism that explains the beneficial effects of exercise on stress-related disorders. The literature from rodent models suggests that the primary benefit of exercise may be the promotion of resilience to stress. The translational value of this hypothesis may be realized through the promotion of exercise as a means to mitigate the longitudinal and cumulative impact of the repeated stressors. Exercise may thus afford both preventative and therapeutic benefits. Given the array of neurotrophins influenced by exercise, the next challenge for future research will be to examine how these varied mechanisms coordinate to optimize neuronal health. The interactions between BDNF and galanin represent an informative example of how trophic mechanisms that mutually promote the maintenance and survival of neurons may require reciprocal regulatory influences to achieve their ultimate benefit.

\section{REFERENCES}

1. Cooney G, Dwan K, Greig C, Lawlor D, Rimer J, Waugh F, et al. Exercise for depression. Cochrane Database Syst Rev (2013) 9:CD004366. doi:10.1002/ 14651858.CD004366.pub6

2. Dunn AL, Trivedi MH, Kampert JB, Clark CG, Chambliss HO. Exercise treatment for depression: efficacy and dose response. Am J Prev Med (2005) 28(1):1-8. doi:10.1016/j.amepre.2004.09.003

3. Herring MP, O'Connor PJ, Dishman RK. The effect of exercise training on anxiety symptoms among patients: a systematic review. Arch Intern Med (2010) 170:321-31. doi:10.1001/archinternmed.2009.530

4. Greenwood B, Fleshner M. Exercise, learned helplessness, and the stressresistant brain. Neuromolecular Med (2008) 10(2):81-98. doi:10.1007/s12017008-8029-y
5. Novak C, Burghardt P, Levine J. The use of a running wheel to measure activity in rodents: relationship to energy balance, general activity, and reward. Neurosci Biobehav Rev (2012) 36(3):1001-14. doi:10.1016/j.neubiorev.2011.12.012

6. Sciolino NR, Holmes PV. Exercise offers anxiolytic potential: a role for stress and brain noradrenergic-galaninergic mechanisms. Neurosci Biobehav Rev (2012) 36:1965-84. doi:10.1016/j.neubiorev.2012.06.005

7. Holmes PV. Rodent models of depression: reexamining validity without anthropomorphic inference. Crit Rev Neurobiol (2003) 15:143-74. doi:10.1615/ CritRevNeurobiol.v15.i2.30

8. Arunrut T, Alejandre H, Chen M, Cha J, Russo-Neustadt A. Differential behavioral and neurochemical effects of exercise, reboxetine and citalopram with the forced swim test. Life Sci (2009) 84(17-18):584-9. doi:10.1016/j.lfs. 2009.02.005

9. Russo-Neustadt A, Ha T, Ramirez R, Kesslak J. Physical activity-antidepressant treatment combination: impact on brain-derived neurotrophic factor and behavior in an animal model. Behav Brain Res (2001) 120(1):87-95. doi:10. 1016/S0166-4328(00)00364-8

10. Duman R, Aghajanian G. Synaptic dysfunction in depression: potential therapeutic targets. Science (2012) 338(6103):68-72. doi:10.1126/science.1222939

11. Ota K, Duman R. Environmental and pharmacological modulations of cellular plasticity: role in the pathophysiology and treatment of depression. Neurobiol Dis (2013) 57:28-37. doi:10.1016/j.nbd.2012.05.022

12. Lehmann M, Herkenham M. Environmental enrichment confers stress resiliency to social defeat through an infralimbic cortex-dependent neuroanatomical pathway. J Neurosci (2011) 31(16):6159-73. doi:10.1523/JNEUROSCI.0577-11.2011

13. Amat J, Baratta M, Paul E, Bland S, Watkins L, Maier S. Medial prefrontal cortex determines how stressor controllability affects behavior and dorsal raphe nucleus. Nat Neurosci (2005) 8(3):365-71. doi:10.1038/nn1399

14. Alleva E, Francia N. Psychiatric vulnerability: suggestions from animal models and role of neurotrophins. Neurosci Biobehav Rev (2009) 33(4):525-36. doi:10.1016/j.neubiorev.2008.09.004

15. Duman R, Monteggia L. A neurotrophic model for stress-related mood disorders. Biol Psychiatry (2006) 59(12):1116-27. doi:10.1016/j.biopsych.2006. 02.013

16. McEwen B. Physiology and neurobiology of stress and adaptation: central role of the brain. Physiol Rev (2007) 87(3):873-904. doi:10.1152/physrev.00041.2006

17. Maier SF, Watkins LR. Role of the medial prefrontal cortex in coping and resilience. Brain Res (2010) 1355:52-60. doi:10.1016/j.brainres.2010.08.039

18. Miguel-Hidalgo J, Whittom A, Villarreal A, Soni M, Meshram A, Pickett J, et al. Apoptosis-related proteins and proliferation markers in the orbitofrontal cortex in major depressive disorder. J Affect Disord (2014) 158:62-70. doi:10.1016/j. jad.2014.02.010

19. Price J, Drevets W. Neurocircuitry of mood disorders. Neuropsychopharmacology (2010) 35(1):192-216. doi:10.1038/npp.2009.104

20. Radley J, Anderson R, Hamilton B, Alcock J, Romig-Martin S. Chronic stressinduced alterations of dendritic spine subtypes predict functional decrements in an hypothalamo-pituitary-adrenal-inhibitory prefrontal circuit. J Neurosci (2013) 33(36):14379-91. doi:10.1523/JNEUROSCI.0287-13.2013

21. Rajkowska G, Miguel-Hidalgo J, Wei J, Dilley G, Pittman S, Meltzer H, et al. Morphometric evidence for neuronal and glial prefrontal cell pathology in major depression. Biol Psychiatry (1999) 45(9):1085-98. doi:10.1016/S0006-3223(99) 00041-4

22. Murray PS, Holmes PV. An overview of brain-derived neurotrophic factor and implications for excitotoxic vulnerability in the hippocampus. Int J Pept (2011) 2011:12. doi:10.1155/2011/654085

23. Stranahan A, Lee K, Martin B, Maudsley S, Golden E, Cutler R, et al. Voluntary exercise and caloric restriction enhance hippocampal dendritic spine density and BDNF levels in diabetic mice. Hippocampus (2009) 19(10):951-61. doi:10.1002/hipo.20577

24. Vaynman S, Zing L, Gomez-Pinilla F. Interplay between BDNF and signal transduction modulators in the regulation of the effects of exercise on synaptic plasticity. Neuroscience (2003) 122:647-57. doi:10.1016/j.neuroscience.2003.08.001

25. Li Y, Luikart B, Birnbaum S, Chen J, Kwon C, Kernie S, et al. TrkB regulates hippocampal neurogenesis and governs sensitivity to antidepressive treatment. Neuron (2008) 59(3):399-412. doi:10.1016/j.neuron.2008.06.023

26. van Praag H, Christie BR, Sejnowski TJ, Gage FH. Running enhances neurogenesis, learning, and long-term potentiation in mice. Proc Natl Acad Sci U S A (1999) 96(23):13427-31. doi:10.1073/pnas.96.23.13427 
27. Adlard P, Cotman C. Voluntary exercise protects against stress-induced decreases in brain-derived neurotrophic factor protein expression. Neuroscience (2004) 124(4):985-92. doi:10.1016/j.neuroscience.2003.12.039

28. Neeper S, Gómez-Pinilla F, Choi J, Cotman C. Exercise and brain neurotrophins. Nature (1995) 373(6510):109. doi:10.1038/373109a0

29. Duman C, Schlesinger L, Russell D, Duman R. Voluntary exercise produces antidepressant and anxiolytic behavioral effects in mice. Brain Res (2008) 1199:148-58. doi:10.1016/j.brainres.2007.12.047

30. van Praag H. Neurogenesis and exercise: past and future directions. Neuromolecular Med (2008) 10(2):128-40. doi:10.1007/s12017-008-8028-z

31. Groves J. Is it time to reassess the BDNF hypothesis of depression? Mol Psychiatry (2007) 12(12):1079-88. doi:10.1038/sj.mp.4002075

32. Petrik D, Lagace D, Eisch A. The neurogenesis hypothesis of affective and anxiety disorders: are we mistaking the scaffolding for the building? Neuropharmacology (2012) 62(1):21-34. doi:10.1016/j.neuropharm.2011.09.003

33. Fuss J, Ben Abdallah NM, Hensley FW, Weber KJ, Hellweg R, Gass P. Deletion of running-induced hippocampal neurogenesis by irradiation prevents development of an anxious phenotype in mice. PLoS One (2010) 5(9):e12769. doi:10.1371/journal.pone.0012769

34. Fuss J, Ben Abdallah NM, Vogt MA, Touma C, Pacifici PG, Palme R, et al. Voluntary exercise induces anxiety-like behavior in adult $\mathrm{C} 57 \mathrm{BL} / 6 \mathrm{~J}$ mice correlating with hippocampal neurogenesis. Hippocampus (2010) 20:364-76. doi:10.1002/hipo.20634

35. Greenwood B, Strong P, Foley T, Thompson R, Fleshner M. Learned helplessness is independent of levels of brain-derived neurotrophic factor in the hippocampus. Neuroscience (2007) 144(4):1193-208. doi:10.1016/j. neuroscience.2006.11.007

36. Hobson SA, Bacon A, Elliot-Hunt CR, Holmes FE, Kerr NC, Pope R, et al. Galanin acts as a trophic factor to the central and peripheral nervous systems. EXS (2010) 102:25-38. doi:10.1007/978-3-0346-0228-0_3

37. Hökfelt T, Tatemoto K. Galanin: a multitalented neuropeptide. EXS (2010) 102:1-5. doi:10.1007/978-3-0346-0228-0_1

38. Holmes PV, Crawley JN. Coexisting neurotransmitters in noradrenergic neurons. In: Bloom FE, Kupfer DJ, editors. Psychopharmacology: The Fourth Generation of Progress. New York: Raven Press (1995). p. 347-53.

39. Hawes J, Picciotto M. Characterization of GalR1, GalR2, and GalR3 immunoreactivity in catecholaminergic nuclei of the mouse brain. J Comp Neurol (2004) 479(4):410-23. doi:10.1002/cne.20329

40. O'Donnell D, Ahmad S, Wahlestedt C, Walker P. Expression of the novel galanin receptor subtype GALR2 in the adult rat CNS: distinct distribution from GALR1. J Comp Neurol (1999) 409(3):469-81. doi:10.1002/(SICI)1096-9861(19990705) 409:3<469::AID-CNE10>3.3.CO;2-H

41. Yoshitake S, Kuteeva E, Hökfelt T, Mennicken F, Theodorsson E, Yamaguchi M, et al. Correlation between the effects of local and intracerebroventricular infusions of galanin on 5-HT release studied by microdialysis, and distribution of galanin and galanin receptors in prefrontal cortex, ventral hippocampus, amygdala, hypothalamus, and striatum of awake rats. Synapse (2014) 68(5):179-93. doi: $10.1002 /$ syn. 21730

42. Sciolino NR, Dishman RK, Holmes PV. Voluntary exercise offers anxiolytic potential and amplifies galanin gene expression in the locus coeruleus of the rat. Behav Brain Res (2012) 233:191-200. doi:10.1016/j.bbr.2012.05.001

43. Murray PS, Groves JL, Pettett BJ, Britton SL, Koch LG, Dishman RK, et al. Locus coeruleus galanin expression is enhanced after exercise in rats selectively bred for high capacity for aerobic activity. Peptides (2010) 31:2264-8. doi:10.1016/j.peptides.2010.09.005

44. Van Hoomissen JD, Holmes PV, Zellner AS, Poudevigne AM, Dishman RK. The effect of B-adrenergic blockade during chronic exercise on contextual fear conditioning and mRNA for galanin and brain-derived neurotrophic factor. Behav Neurosci (2004) 118:1378-90. doi:10.1037/0735-7044.118.6.1378

45. Chambliss HO, Van Hoomissen JD, Holmes PV, Bunnell BN, Dishman RK. Effects of chronic activity wheel running and imipramine on male copulatory behavior after olfactory bulbectomy. Physiol Behav (2004) 82:593-600. doi:10.1016/j.physbeh.2004.04.064

46. Holmes PV, Yoo HS, Dishman RK. Voluntary exercise and clomipramine treatment elevate prepro-galanin mRNA levels in the locus coeruleus in rats. Neurosci Lett (2006) 408:1-4. doi:10.1016/j.neulet.2006.04.057

47. Karlsson RM, Holmes A. Galanin as a modulator of anxiety and depression and a therapeutic target for affective disease. Amino Acids (2006) 31(3):231-9. doi:10.1007/s00726-006-0336-8
48. Lu X, Ross B, Sanchez-Alavez M, Zorrilla E, Bartfai T. Phenotypic analysis of GalR2 knockout mice in anxiety- and depression-related behavioral tests. Neuropeptides (2008) 42(4):387-97. doi:10.1016/j.npep.2008.04.009

49. Le Maître T, Xia S, Le Maitre E, Dun X, Lu J, Theodorsson E, et al. Galanin receptor 2 overexpressing mice display an antidepressive-like phenotype: possible involvement of the subiculum. Neuroscience (2011) 190:270-88. doi:10. 1016/j.neuroscience.2011.05.015

50. Hobson S, Bacon A, Elliot-Hunt C, Holmes F, Kerr N, Pope R, et al. Galanin acts as a trophic factor to the central and peripheral nervous systems. Cell Mol Life Sci (2008) 65(12):1806-12. doi:10.1007/s00018-008-8154-7

51. Hobson S, Vanderplank P, Pope R, Kerr N, Wynick D. Galanin stimulates neurite outgrowth from sensory neurons by inhibition of Cdc42 and Rho GTPases and activation of cofilin. JNeurochem (2013) 127(2):199-208. doi:10.1111/jnc.12379

52. Chen Y, Kramár E, Chen L, Babayan A, Andres A, Gall C, et al. Impairment of synaptic plasticity by the stress mediator $\mathrm{CRH}$ involves selective destruction of thin dendritic spines via RhoA signaling. Mol Psychiatry (2013) 18(4):485-96. doi:10.1038/mp.2012.17

53. Pirondi S, Fernandez M, Schmidt R, Hökfelt T, Giardino L, Calzà L. The galanin-R2 agonist AR-M1896 reduces glutamate toxicity in primary neural hippocampal cells. J Neurochem (2005) 95(3):821-33. doi:10.1111/j.1471-4159. 2005.03437.x

54. Koleske A. Molecular mechanisms of dendrite stability. Nat Rev Neurosci (2013) 14(8):536-50. doi:10.1038/nrn3486

55. Binder DK, Croll SD, Gall CM, Scharfman HE. BDNF and epilepsy: too much of a good thing? Trends Neurosci (2001) 24(1):47-53. doi:10.1016/S0166-2236(00) 01682-9

56. Papaleo F, Silverman JL, Aney J, Tian Q, Barkan CL, Chadman KK, et al. Working memory deficits, increased anxiety-like traits, and seizure susceptibility in BDNF overexpressing mice. Learn Mem (2011) 18(8):534-44. doi:10.1101/lm.2213711

57. Scharfman HE, Goodman JH, Sollas AL, Croll SD. Spontaneous limbic seizures after intrahippocampal infusion of brain-derived neurotrophic factor. Exp Neurol (2002) 174:201-14. doi:10.1006/exnr.2002.7869

58. Ramsden M, Berchtold NC, Kesslak JP, Cotman CW, Pike CJ. Exercise increases the vulnerability of rat hippocampal neurons to kainate lesion. Brain Res (2003) 971(2):239-44. doi:10.1016/S0006-8993(03)02365-5

59. Reiss JI, Dishman RK, Boyd H, Robinson JK, Holmes PV. Chronic activity wheel running reduces the severity of kainic acid-induced seizures in the rat: possible role of galanin. Brain Res (2009) 1266:54-63. doi:10.1016/j.brainres. 2009.02.030

60. Mazarati A, Langel U, Bartfai T. Galanin: an endogenous anticonvulsant? Neuroscientist (2001) 7(6):506. doi:10.1177/107385840100700607

61. Mazarati A, Lu X. Regulation of limbic status epilepticus by hippocampal galanin type 1 and type 2 receptors. Neuropeptides (2005) 39(3):277-80. doi:10.1016/j.npep.2004.12.003

62. Melander T, Hökfelt T, Rökaeus A, Cuello A, Oertel W, Verhofstad A, et al. Coexistence of galanin-like immunoreactivity with catecholamines, 5hydroxytryptamine, GABA and neuropeptides in the rat CNS. J Neurosci (1986) 6(12):3640-54.

63. Lu X, Sharkey L, Bartfai T. The brain galanin receptors: targets for novel antidepressant drugs. CNS Neurol Disord Drug Targets (2007) 6(3):183-92. doi:10.2174/187152707780619335

Conflict of Interest Statement: The author declares that the research was conducted in the absence of any commercial or financial relationships that could be construed as a potential conflict of interest.

Received: 21 May 2014; accepted: 14 July 2014; published online: 28 July 2014. Citation: Holmes PV (2014) Trophic mechanisms for exercise-induced stress resilience: potential role of interactions between BDNF and galanin. Front. Psychiatry 5:90. doi: $10.3389 /$ fpsyt.2014.00090

This article was submitted to Affective Disorders and Psychosomatic Research, a section of the journal Frontiers in Psychiatry.

Copyright (C) 2014 Holmes. This is an open-access article distributed under the terms of the Creative Commons Attribution License (CC BY). The use, distribution or reproduction in other forums is permitted, provided the original author(s) or licensor are credited and that the original publication in this journal is cited, in accordance with accepted academic practice. No use, distribution or reproduction is permitted which does not comply with these terms. 